\title{
HoloVizio 3D Display System
}

\author{
Tibor Balogh \\ Holografika
}

t.balogh@holografika.com

\author{
Péter Tamás Kovács \\ Holografika \\ Baross str. 3. Budapest \\ $\mathrm{H}-1192$, Hungary \\ +3612824921
}

p.kovacs@holografika.com
Zoltán Megyesi
Holografika

\section{z.megyesi@holografika.com}

\begin{abstract}
3D displaying can be crucial in many modern applications that use 3D models, volumes or otherwise benefit from 3D information. Also, future visual entertainment can not stop at 2D displaying. In this paper, we describe the HoloVizio true 3D display system. The patented HoloVizio technology uses a specially arranged array of optical modules and a holographic screen. Each point of the holographic screen emits light beams of different color and intensity to various directions. With proper software control, light beams leaving the pixels propagate in multiple directions, as if they were emitted from the points of $3 \mathrm{D}$ objects at fixed spatial locations. We show that the direction selective light emission is a general requirement for every $3 \mathrm{D}$ system and the advantages of light field reconstruction over the multiview approach. We describe the 10 Mpixel desktop display and the 50Mpixel large-scale system. We cover the real-time control issues at high pixel-count systems with the HoloVizio software environment, give solutions for natural content displaying, and describe concrete 3D applications developed in the frame of European projects.
\end{abstract}

\section{Keywords}

3D display, 3DTV, holography, stereoscopic, autostereoscopic, multiview, projection, microdisplay

\section{INTRODUCTION}

Displaying 3D images is a major step towards realistic visualization. Providing perceivable 3D information can be crucial in applications of many areas including architecture design, chemical and biological research, industrial engineering, geological surveying, medical visualization and more. However, displaying 3D images is not an easy task.

A 3D image contains much more information than a corresponding 2D image. To display proper three-dimensional images 3D systems should produce sufficient number of points in

Permission to make digital or hard copies of all or part of this work for personal or classroom use is granted without fee provided that copies are not made or distributed for profit or commercial advantage and that copies bear this notice and the full citation on the first page. To copy otherwise, or republish, to post on servers or to redistribute to lists, requires prior specific permission and/or a fee.

IMMERSCOM 2007, October 10-12, 2007, Verona, Italy.

Copyright 2007 ICST 978-963-9799-06-6

DOI 10.4108/ICST.IMMERSCOM2007.2145 a given time frame, i.e. to provide appropriate pixel/sec rate. This is the TB law (total number of beams). In case the system does not globally have the capability of supplying sufficient number independent spots or pixels per second, the resulting 3D image will not be compromise-free. By this calculation it is possible to determine the quality of any $3 \mathrm{D}$ solution immediately, independently of what is promoted.

Direction selective light emission is the common feature for any 3D system comprising of a screen (this is true for the outer surface of volumetric systems even with exotic arrangements).

In case we would have a light emitting surface, on which each point is capable to emit multiple light beams of various intensity and color to multiple directions in a controlled way, the problem of 3D displaying would be solved and we would have a perfect 3D display.

\section{STATE OF THE ART 3D DISPLAYS}

The most widespread solutions are stereoscopic displays with tracking. Stereoscopic displays [6] can emit only two distinguishable light beams from each pixel, this is the reason for the compromises: the necessity of being positioned at the sweet point, the viewer dependent view, or the necessity of tracking [7] to create motion parallax, which is still for single/few user only and imply inaccuracies and latency.

Multiview systems [5] produce $8-16$ views, which is still strongly compromised causing the jumpy character, limited FOV and invalid zones. Since most of these systems, except time multiplexing solutions, use $2 \mathrm{D}$ panels as the base, there is a tradeoff between directions and resolution: introducing $n$ directions decrease the resolution to the $1 / \mathrm{n}$ part. This is especially true for integral imaging where the vertical views eat up the available resolution. In other words integral imaging is a full version multi-view system with vertical parallax.

Volumetric systems do not follow current displaying conventions, they rather follow the looking into than the looking out philosophy. The same global number applies also to volumetric systems however the number represents the number of addressable spatial spots, while at the other systems the spatial positions are represented by the available combinations of light beams. This leads to the problem of missing occlusion. Light never covers light, bright spots in the back will never disappear and there is no way to show natural scenes with hidden edges, translucent images or wire-frames only. 
Holographic systems generate holographic patterns to reconstruct the wavefront. These systems suffer fundamental limitations on realistically achievable image sizes, viewing angle and it must be noted such systems also do not have infinite resolution in the FOV. Having the same imager as the base, the overall number of spots is the same that can be produced by diffractive or refractive imaging. In practice holographic solutions show poorer performance because of the limited phase modulation capabilities of available components.

\section{COLLABORATIVE 3D MEDICAL APPLICATIONS}

Modern clinical practice is heavily dependent on intrinsically $3 \mathrm{D}$ imaging modalities such as CT, MRI and related technologies. Since the invention of the Marching cubes algorithm, and fast direct volume rendering techniques, it has become common to perform pre-operative studies on virtual models of the affected organs, often using standard stereoscopic technology, e.g., 3D eyeglasses and appropriate head tracking devices. While this approach provides valuable information, it requires a cumbersome, single-viewer work environment that clashes with the modern multi-speciality collaboration that is gradually becoming the preferred approach to the planning of major surgical operation.

The definition of a collaborative 3D environment for clinical applications is a very recent research subject. To date, research is mostly concentrating either on the application of available multiview displays, or the creation of augmented reality systems, based on the duplication of standard stereoscopic technology setup. As has been discussed earlier, the use of the former solution in a collaborative environment is severely hampered by the very small viewing angle of the multi-view displays, while the latter forces the clinicians to wear 3D eyeglasses. A working collaborative 3D medical application should allow the participant to interact with multi-dimensional data, and provide them with a mechanism to directly annotate the volumetric data. How this should be done is still an open research problem.

Current systems take in excess of 48 hours to produce high quality 3D rendered medical data, which can support multi-user viewing of (static) 3D rendered medical data, e.g. VOXEL Digital Holography. These systems interface with existing medical imaging equipment to produce 3D reconstructions stored as holograms on hard-copy films and use a proprietary holographic display system to display the internal patient anatomy. While this method provides an actual high quality $3 \mathrm{D}$ rendering, it requires several days to produce the hard-copy film, and, since it is an essentially analogue in nature, it does not support any kind of real interaction, e.g., annotation, of the data. Given the obvious advantage of maintaining clinical data in digital form, it is clear that this system can provide, at most, a temporary solution to an increasingly pressing problem. Moreover, collaboration among multiple geographically dispersed teams is difficult with this solution. In this context, networked virtual reality systems are considered one of the most promising (and challenging) research areas. Significant and pioneering work has already been done in the area of networked CAVE applications. Developments of such networks will ultimately allow medical teams to share a virtual space, where each team member experiences and interacts with identical environmental and patient scenarios simultaneously, independently from the particular geographic location of their teams.

\section{THE HOLOVIZIO DISPLAY SYSTEM}

The patented HoloVizio technology uses a specially arranged array of optical modules and a holographic screen. Each point of the holographic screen emits light beams of different color and intensity to the various directions. The light beams generated in the optical modules hit the screen points in various angles and the holographic screen makes the necessary optical transformation to compose these beams into a perfectly continuous 3D view, see Fig. 1. With proper software control, light beams leaving the pixels propagate in multiple directions, as if they were emitted from the points of 3D objects at fixed spatial locations. The direction selective light emission is a general requirement for every $3 \mathrm{D}$ system and provides quantitative data on the FOV, on the angular resolution, determining field of depth of the displays, affecting the total number of light beams necessary for high-end 3D displaying.

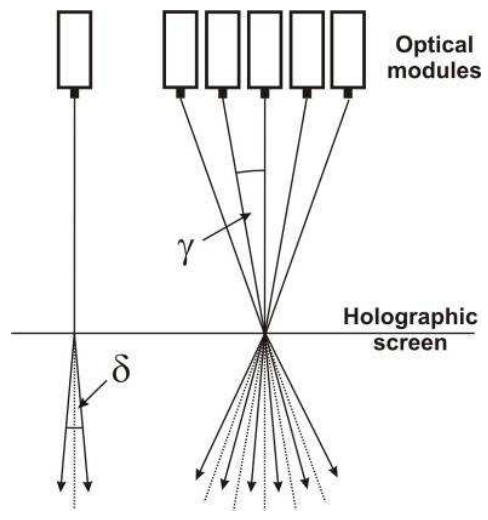

Figure 1. HoloVizio principle

The HoloVizio technology can be used to build both small-scale and large-scale display systems.

In the following we first present the hardware and software components of the 50Mpixel large-scale system, and then the small-scale systems. [3] [4]

\section{LARGE-SCALE HOLOVIZIO SYSTEM}

\subsection{Hardware system}

We developed a 50 Mpixel large-scale system [1] with a screen diagonal above $1,8 \mathrm{~m}$, see Fig. 2. The system has a slightly immersive curved configuration. The optical system consists of compact projection modules, arranged in horizontal rows. The projector modules are densely arranged in horizontal-onlyparallax configuration behind the holographic screen and all of them project their specific image onto the holographic screen. The system has a high angular resolution, and approximately 50 independent light beams originate from each pixel. 


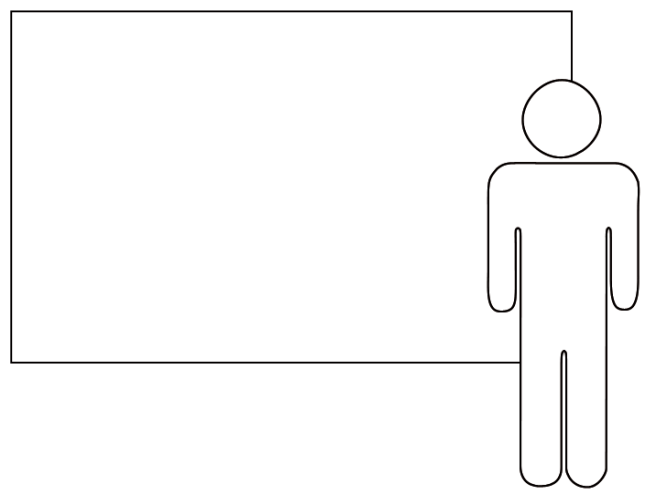

Figure 2. Size of the large-scale system

Considering the 50 Mpixel overall resolution, the 2D equivalent image resolution with conventional terms would correspond to $1344 \times 768$ and such views build up a 3D view with continuous motion parallax.

The display could work at 25 frames/sec refresh rate in color sequential mode, though the refresh rate is different of the frame rate that depends on the user's computing hardware. The display is prepared to work in hybrid mode, that is, it can show exceptional brightness when high brightness is needed, even for outdoor illumination conditions, or it is possible to alter the illumination system working in economic mode as a LED display.

A PC-based render cluster feeds the display with 50 Mpixels in real-time and a sophisticated control system controls the projectors, PC-s, the network, power supplies and monitors all system parameters. The control system architecture consists of two main subsystems. The first is the PC based render cluster of 16 PC-s with dual PCI-Expressx16, with high-end nVidia graphics cards, high-speed dual Gbit ETH network components and the server.

The cluster is responsible for the rendering process and it feeds the display with 50 Mpixels in real-time. The data input is up to a dual Gigabit ETH and according to the measurements it is capable to process 1,3Gigabit/sec with the overheads. As of the further management functions, the second part, a sophisticated control system controls the projectors, PC-s, the network, cooling system, power supplies \& UPS and monitor all system parameters.

With the HoloVizio approach we can build displays that have excellent image resolution of 1920x1080 or beyond, large FOV above 100 degrees, large Field-of-Depth, and at the same time we state the number of pixels being in the range of hundreds of millions. As a result the system architecture it is not limited in any of the general 3D display's parameters; various systems could be achieved for various applications based on the principle.

\subsection{Software system}

Legacy interactive graphics applications are interfaced to the holographic displays through a special implementation of OpenGL, the HoloVizio OpenGL wrapper. This wrapper library intercepts all calls the application sends to OpenGL, but also transmits them to the original OpenGL library transparently, so that the 3D windows of the application will remain the same, see Fig. 3. The rendering process is about generating the set of images to be projected from different positions according to the connected optical modules.

When running in cluster configuration, the wrapper transmits the call stream to the nodes of a render cluster through a DualGigabit Ethernet network, which then render their part and show the resulting images on the display.

Aside from the OpenGL command stream, this image generation algorithm is fed with the optical module geometry (position, frustum) and physical specifications. The latter are used for realtime calibration, to correct geometrical nonlinearities, to adjust color and intensity response and match the screen diffusion capabilities. This comes from pre-recorded data sets obtained through an automated display calibration process. Angular resolution correction (depth dependent anti-aliasing) is performed as well. Calibration is achieved in real time, and has been implemented on the GPUs as vertex and pixel shaders.

In the HoloVizio software system we have additional software utilities for various 3D source formats, to generate 3D images from 2D camera image sequences or video, 2D camera images with depth maps, 3D model, etc. We operate both on Linux and Windows, running on standard PCs.

\section{SMALL-SCALE HOLOVIZIO SYSTEMS}

\subsection{Hardware system}

As of monitor style 3D displays currently we produce two models, a 32", 10 Mpixel, 16:9 aspect and a 26" 7,4 Mpixel 4:3 aspect model [2]. These models are in the dimensions of normal TV sets. The 10 Mpixels and 7,4 Mpixels provided by 128 and 96 LCD microdisplay panels respectively. The modules contain the fast LCD panels and dedicated plastic aspheric optics. The optical modules are densely arranged in horizontal-only-parallax configuration behind the holographic screen and all of them project their specific image onto the holographic screen. The imaging optics of the modules have a wide angle, which results in a 50 degrees FOV and the angular resolution of the displays is 0.8 degrees. This means that $\sim 60$ independent light beams originate from each pixel in this FOV.

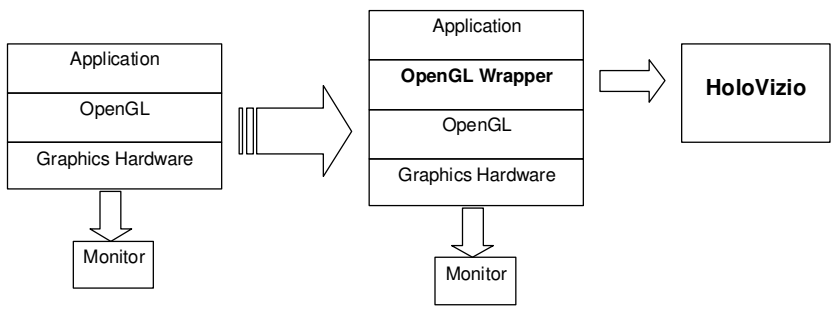

Figure 3. The wrapper is a layer between OpenGL and the application

At the 10Mpixel display with conventional terms this would correspond to 60 views, each of $512 \times 320$. It fits quite well certain applications like medical, where the CT, MR DICOM datasets are of $512 \times 512$ resolution. If we multiply the numbers, 
this is what 10 million pixels can back-up in 50 degrees FOV and a FOD in the range of the screen size volume. In subjective tests however viewers estimate better (approx. a normal TV) resolution, because the brain integrate the details from the eyes and spontaneous movements. As a consequence of the technology the displays can show 2D without the need of switch through, thus providing a smooth compatibility and coexistence with conventional 2D TV systems. The displays work at a high 50 frames/sec refresh rate in color sequential mode. The refresh rate is different from the frame rate that depends on the user's computing hardware.

The displays have 4 DVI inputs to support the required bandwidth. A high-speed image-processing unit decodes the input DVI streams. The DVI channels work at $1280 \times 1024$ at $60 / 75 \mathrm{~Hz}$ and are thus capable of transmitting up to $280 \mathrm{MB} / \mathrm{sec}$ per channel. Due to the flexible system architecture it is possible to use the displays with $1,2,3$ or 4 DVI channels. The user can use a PC with standard 3D graphics cards and depending on the number of DVI outputs used, the displays will work at different frame rates. By one or two DVI channel 8 or 16 frames/sec can be reached respectively. An uncompressed 3D image for the 10Mpixel display is $30 \mathrm{MB}$, thus $3 \mathrm{DVI}$ channels are sufficient for the 25 frame/sec real-time operation. A typical configuration is the dual display mode, using 3 DVI channels for the HoloVizio and 1 for a 2D display.

\subsection{Software system}

As for the large-scale system, we have an OpenGL wrapper for driving the small-scale displays. In this case, there is no need to use a rendering cluster, the display is driven by a single PC, the same that runs the user's application. One or two video cards (including the one that renders the application and the desktop itself) are used to render several pictures from different points of view to an off-screen surface, which are then transferred to the display device through DVI connector(s). The same imageenhancing techniques (calibration, anti-aliasing) described at the large-scale system are performed on this platform too.

\section{Natural content}

To display natural content on a $3 \mathrm{D}$ display, it is not enough to show an image recorded by a single conventional camera. Additional sources are needed to provide 3D information. For large FOV displays like the HoloVizio systems, several cameras are needed to capture natural scenes, and they must be placed so that to provide information from a large FOV. Depending on the number of cameras, different algorithms exist to extract 3D information from the camera images. If the number of cameras is high (70-100), the light rays necessary for composing 3D scenes can be calculated using interpolation methods also used for Light Field Rendering [8]. If the number of cameras is lower (7-11), depth information must be calculated and intermediate views must be rendered $[9,10]$. This, however is a computation costly process and must be performed off line. Figure 4 illustrates natural content visualized on the Large Scale HoloVizio System. The 100 frames Breakdancers dataset [11] has been recorded by 8 cameras and depth information was calculated for each image in each frame [9].

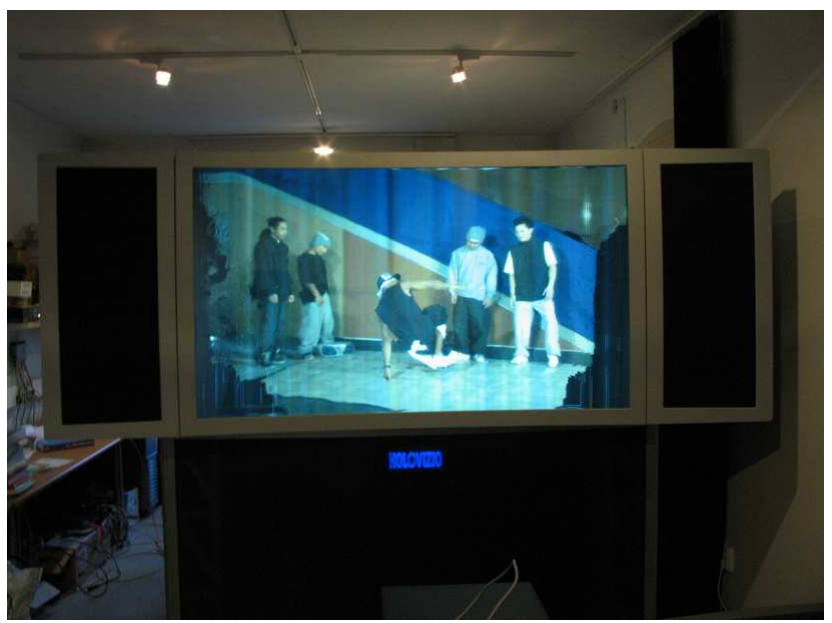

Figure 4.: Natural 3D content displayed on the large-scale HoloVizio system

\section{APPLICATIONS AND FUTURE WORK}

Holografika is involved in several R\&D projects in the frame of the EU FP6 and national programs, targeting next generation 3D displays and novel 3D applications. Among these we are developing displays above $125 \mathrm{Mpixels}$ based on the very latest microdisplay and LED technology. The FP6 project OSIRIS aims to create novel display systems including a high resolution LED based compact display that is capable of real time processing and playing of live captured natural content. In the same project the HoloVizio technology is applied in an even larger scale, to create a 3D Cinema application. The earlier 50Mpixel system was developed for automotive application, and was tested by a major European car manufacturer in selected use scenarios in the car design process, where each viewer see the 3D view simultaneously with correct positions enabling collaborative work without glasses or other supplementary devices freeing up the viewer's hands and significantly enhancing collaborative interactive medical work while investigating 3D images originating from CT, MR, PET, 3D Ultrasound or other suitable medical applications.

The wider scope is to develop a networked holographic audiovisual platform to support multi-user real-time collaborative $3 \mathrm{D}$ interaction between geographically distributed teams. In the frame of the project together with European partners we develop additional system components, such as a camera based gesture recognition system to intuitively interact with the $3 \mathrm{D}$ objects in the displayed scene.

Using the OpenGL wrapper technology, we have successfully visualized scenes from numerous existing software packages, including $\mathrm{CAD}$, medical and oil-industry related software in real time.

Further systems are under development for air-traffic control, architectural and terrain visualization, virtual theatre and telepresence applications. In the frame of a national project we performed 3D image transmission under standard DVB-C platform and development work is in progress on complete $3 \mathrm{D}$ 
chain, including multi-camera 3D acquisition, compression and storage beyond the displaying.

\section{CONCLUSIONS}

We explained that $3 \mathrm{D}$ systems must use more pixels or higher speed components than current $2 \mathrm{D}$ displays and that the direction selective light emission is a common requirement for $3 \mathrm{D}$ displays. Based on this we characterized known 3D solution categories pointing the limitations. We described the HoloVizio 128WD and 96ND desktop displays and the 50Mpixel largescale HoloVizio 640RC system that are on the market and are used in various applications.

3D displays cannot be considered anymore as just toys or novelties. The 3D data is widely used and there is an obvious need for 3D displays. The major obstacle of being accepted up to now is the fact that most of them were simply not good enough for real applications. On the long term, particularly in the 3D television only such a system will survive that does not make any inconvenience, side effects to the viewers and provide the same freedom as $2 \mathrm{D}$ systems. The technology is here and the $3 \mathrm{D}$ is evidently the next step in the displaying. While the transition from black\&white to color TV represents a factor of $3 x$ increase in the information content, HDTV represent a factor of 4-5, true 3D displaying requires orders of magnitudes, 102 to 104 boost in the display capabilities and thus it will be the most significant move, almost a paradigm shift, in the display and television technologies and the related environment for the coming years.

\section{ACKNOWLEDGMENTS}

This work has been supported by EU IST-FP6 Integrated Project OSIRIS (IST-33799 IP).

\section{REFERENCES}

[1] T. Balogh, T. Forgacs, T. Agocs, E. Bouvier, F. Bettio, E. Gobbetti and G. Zanetti: A Large Scale Interactive Holographic Display. IEEE VR2006 Conference, March 2529, Alexandria, Virginia, USA

[2] T. Balogh, T. Forgacs, T. Agocs, O. Balet, E. Bouvier, F. Bettio, E. Gobbetti and G. Zanetti: A Scalable Hardware and Software System for the Holographic Disp of Interactive Graphic Applications. EuroGraphics 2005, Dublin, Aug. 2005

[3] T. Balogh "Method and apparatus for displaying threedimensional images", U.S. Patent 6,201,565, EP 0900501, Feb 04, 1997 [HU]

[4] T. Balogh "Method and apparatus for displaying 3d images", U.S. Patent Appl. 20030156077, EP 1 285304, May 19, 2000 [HU]

[5] Cees van Berkel, David W Parker and Anthony R. Franklin: Multiview 3D-LCD. In Stereoscopic Displays and Virtual Reality Systems III (1996), Vol. 2653 of SPIE proceedings, pp.32

[6] D. Ezra, G. J. Woodgate, B. A. Omar, N. S. Holliman, J. Harrold and L. S. Shapiro: New autostereoscopic display system. In Stereoscopic Displays and Virtual Reality Systems II (1995), Vol. 2409 of SPIE proceedings, pp.31-40

[7] G. J. Woodgate, D. Ezra, J. Harrold, Nicolas S. Holliman, G. R. Jones, R. R. Moseley: Autostereoscopic 3D display systems with observer tracking. In Image Communication Special Issue on 3D Video Technology (EURASIP - 1998), pp. 131

[8] M. Levoy, and P. Hanrahan, "Light Field Rendering", Proc. ACM SIGGRAPH, pp. 31-42, August 1996.

[9] C.L. Zitnick, S.B. Kang, M. Uyttendaele, S. Winder, and R. Szeliski, "High-quality video view interpolation using a layered representation” ACM SIGGRAPH and ACM Trans. on Graphics, Los Angeles, CA, Aug. 2004, pp. 600-608.

[10] A.W. Fitzgibbon, Y. Wexler, A. Zisserman, "Image-based rendering using image-based priors”, Proc. International Conference on Computer Vision, October 2003.

[11] http://research.microsoft.com/vision/InteractiveVisualMedia Group/3DVideoDownload/ 